\title{
DIFERENCIAS DE PERFECCIONISMO EN FUNCIÓN DEL GRADO DE RECHAZO ESCOLAR POR ESCAPAR DE LA AVERSIÓN SOCIAL O SITUACIONES DE EVALUACIÓN: ANÁLISIS EN POBLACIÓN JUVENIL DE ECUADOR
}

\author{
Ma Pilar Aparicio Flores \\ Universidad de Alicante \\ pilar.aparicio@ua.es \\ Virginia Narcisa Ortega Sandoval \\ Universidad Central del Ecuador \\ Aitana Fernández Sogorb \\ Universidad de Alicante \\ José Manuel García Fernández \\ Universidad de Alicante
}

Fecha de Recepción: 2 Septiembre 2019

Fecha de Admisión: 25 Septiembre 2019

\begin{abstract}
RESUMEN
Una de las características de los seres humanos es desarrollarse hasta alcanzar metas y objetivos personales. Sin embargo, los logros alcanzados en muchos de los casos son evaluados y asociados como triunfos personales, pero existe un grupo de individuos que sus autoevaluaciones son demasiadas rígidas, y piensan que no lo hicieron lo suficientemente bien, ocasionándoles insatisfacciones personales; son aquellos que se denominan perfeccionistas. A su vez, existe un colectivo de población infanto-juvenil que rechaza ir a la escuela debido a esas dificultades ante situaciones de evaluación y de exponer en público. Es decir, por la aversión social o situaciones de evaluación académica. Por ello, el presente trabajo tuvo como objetivo estudiar las diferencias estadísticamente significativas de perfeccionismo en estudiantes ecuatorianos en función de las altas y bajas puntuaciones en el escape de la aversión social o situaciones de evaluación, cuyo factor corresponde a la II dimensión de la School Refusal Assessment Scale-Revised for Children (SRAS-R-C). La muestra total alcanzó un total de 1920 estudiantes de 1ํㅜㄴ $2^{\circ}$ y $3^{\circ}$ de Bachillerato matriculados en institutos públicos de Ecuador. Los hallazgos observados arrojan diferencias estadísticamente significativas en ambos grupos para variables como Perfeccionismo Auto Orientado Esfuerzos (PAO-E) y Perfeccionismo Auto Orientado Crítica (PAO-C), siendo los adolescentes con alto rechazo escolar los que obtuvieron más altas puntuaciones en PAO-C $(d=.18)$ y PAO-E $(d=.27)$. En conclusión, los hallazgos encontrados suponen un avance científico en el ámbito educativo, teniendo en cuenta la importancia de no abandonar la vida académica para el futuro laboral, económico y social del país de Ecuador.
\end{abstract}


Palabras clave: perfeccionismo auto orientado esfuerzos; perfeccionismo auto orientado crítica; rechazo escolar; aversión social; adolescencia.

\section{ABSTRACT}

Perfectionism differences depending on the level of school refusal for escaping the social aversion or evaluation situations: analysis in Ecuador youth population. One of the characteristics of human beings is to develop to achieve personal goals and objectives. However, the achievements made in many cases are evaluated and associated as personal triumphs, but there is a group of individuals whose self-assessments are too rigid, and think they did not do well enough, causing them personal dissatisfaction. They are those that are called perfectionists. In turn, there is a group of children and young people who refuse to go to school due to these difficulties in situations of evaluation and public presentation. That is, by escape from aversive social and/or evaluative situations. Therefore, the present work aimed to study the statistically significant differences of perfectionism in Ecuadorian students based on high and low scores in the escape from aversive social and/or evaluative situations, whose factor corresponds to the II dimension of the Refusal School Assessment Scale-Revised for Children (SRAS-RC). The total sample reached a total of 1920 students of 1st, 2nd and 3rd Baccalaureate enrolled in public institutes of Ecuador. The observed findings show statistically significant differences in both groups for variables such as Self-Oriented PerfectionismStrivings (SOP-S) and Self-Oriented Perfectionism-Critical (SOP-C), with adolescents with high school rejection those who obtained higher scores in SOP-C $(d=.18)$ and SOP-S $(d=.27)$. In conclusion, the findings found represent a scientific advance in the educational field, taking into account the importance of not abandoning academic life for the labor, economic and social future of the country of Ecuador.

Keywords: self-oriented perfectionism-strivings; self oriented perfectionism-critical; school refusal; social aversion; adolescence.

\section{INTRODUCCIÓN}

A lo largo de los últimos años, el estudio sobre el Perfeccionismo va en aumento. Hamachek (1978) y Burns (1980) fueron de los iniciantes que aportaron soporte teórico al constructo, con una de las definiciones más acertadas. En este sentido, Burns, Io definió como "la aplicación de altos estándares más allá del alcance o la razón, el esfuerzo compulsivo e incesante hacia metas imposibles y la evaluación del propio valor exclusivamente en términos de productividad y logro" (1980; p. 11).

Estos aportes son apoyados por diversos estudios especializados en el constructo, los cuales se dividen entre aquellos que piensan que hay un perfeccionismo sano 0 adaptativo, y otro insano 0 desadaptativo (Rice, Bair, Castro, Cohen y Hood, 2003; Stoeber y Otto, 2006). A este respecto, algunos estudios manifiestan que existen sujetos con un perfeccionismo que no es perjudicial para la persona, fruto de una alta responsabilidad y de una gran motivación y placer por ejecutar positivamente sus tareas, el cual lleva a planificar los proyectos y a cumplirlos eficazmente. Es decir, son individuos que realizan los actos por satisfacción personal y no para causar una impresión correcta (Slade y Owens, 1998).

No obstante, por el lado contrario, autores como Flett y Hewitt (2005) revelan un segundo perfil de perfeccionismo patológico. Este tipo de perfeccionismo, según los autores, se da cuando las actividades se ejecutan con presión por uno mismo, teniendo en cuenta que se desea alcanzar niveles tan altos de perfección que les lleva a estados emocionales negativos y pensamientos patológicos, tales como la ansiedad (Inglés, García-Fernández, Vicent, Gonzálvez y Sanmartín, 2016). De hecho, es predictor de un alto rechazo a la escuela, sobre todo debido a ese rechazo para evitar 
situaciones sociales aversivas y/o en las que se sienten evaluados (Gonzálvez, Sanmartín, GarcíaFernández, Inglés y Vicent, 2016), segundo de los factores de la School Refusal Assessment ScaleRevised for Children (Kearney, 2002; Gonzálvez et al., 2016).

Ya las primeras investigaciones de rechazo a la escuela, por Jung (1913) definían esta problemática como un rechazo neurótico por parte del niño hacia a asistir a la institución educativa. Investigaciones posteriores, lo denominaron como un miedo infantil (Broadwin, 1932) una fobia escolar (Johson, Falstein, Szurek y Svendsen, 1941) o una ansiedad por separación (Ester, Haylett y Johnson, 1956).

En la actualidad, perdura la definición de Kearney y Bates (2005) cuyos autores la definen como una dificultad de permanecer 0 acudir a la escuela que persiste en niños y jóvenes. Y dentro de esta conceptualización, cuatro son las dimensiones que caracterizan a la variable; entre las que se encuentra ese rechazo para evitar situaciones sociales aversivas y/o en las que se sienten evaluados. Es decir, esa aversión social o esas dificultades ante situaciones de evaluación o de exponer en público.

El conocimiento científico de este constructo va en aumento, siendo los principales temas de estudio además de su delimitación conceptual, los instrumentos de medida, tratamientos, comorbilidad, absentismo y factores de riesgo del mismo (García-Fernández et al., 2016). Sin embargo, y a pesar de que es una temática de estudio a nivel mundial, debido a sus características y las consecuencias que ello conlleva en la futura sociedad, en el país de Ecuador las investigaciones sobre rechazo escolar son casi inexistentes. Y es por ello, que el propósito de este estudio es identificar la relación entre el perfeccionismo y el rechazo escolar, concretamente, de la evitación de situaciones sociales aversivas y/o en las que se sienten evaluados. A este respecto, cabe destacar, según Vicent (2017) y Ortega-Sandoval (2019) que el perfeccionismo se conceptualiza de tres dimensiones que componen un Perfeccionismo Socialmente Prescrito (PSP), basado en el pensamiento de que las personas significativas esperan que uno mismo alcance la perfección; un Perfeccionismo Auto Orientado-Esfuerzos (PAO-E), el cual contempla pretensiones y esfuerzos por ser perfecto; y un Perfeccionismo Auto Orientado-Crítica (PAO-C), el cual lleva consigo autocríticas y miedo por caer en el error.

\section{OBJETIVOS DE LA INVESTIGACIÓN}

El objetivo del presente trabajo trata de observar si existen diferencias estadísticamente significativas en PSP, PAO-C y PAO-E en función de si los adolescentes examinados presentan un alto 0 bajo nivel del segundo de los factores del SRAS-R-C, "escapar de la aversión social o situaciones de evaluación".

\section{MUESTRA Y/O PARTICIPANTES}

El número total de participantes entrevistados fue de 1786 alumno/as del país de Ecuador, matriculados en 1ํㅜㄴ $2^{\circ}$ y $3^{\circ}$ de Bachillerato. Esta muestra total quedó compuesta de este modo después de excluir a 134 participantes por omisiones en sus respuestas o no presentar el consentimiento informado (véase Tabla 1).

Como es posible observar en la Tabla 1, la muestra participante comprendía edades entre 15 y 18 años ( $\left.M_{\text {edad }}=16.31 ; D E=1.00\right)$, y un 49\% pertenecían al sexo femenino. 
DIFERENCIAS DE PERFECCIONISMO EN FUNCIÓN DEL GRADO DE RECHAZO ESCOLAR POR ESCAPAR DE LA AVERSIÓN SOCIAL O SITUACIONES DE EVALUACIÓN: ANÁLISIS EN POBLACIÓN JUVENIL DE ECUADOR

Tabla 1.

Tabla de contingencia sexo*curso con frecuencias y porcentajes

\begin{tabular}{ccccc}
\hline & \multicolumn{3}{c}{ Curso } & Total \\
\hline Sexo & $1^{\mathrm{o}}$ & $2^{\mathrm{o}}$ & $3^{\mathrm{o}}$ & \\
Chicos & 366 & 308 & 236 & 910 \\
$\%$ & $20.5 \%$ & $17.2 \%$ & $13.2 \%$ & $51.0 \%$ \\
Chicas & 342 & 296 & 238 & 876 \\
$\%$ & $19.1 \%$ & $16.6 \%$ & $13.3 \%$ & $49.0 \%$ \\
Total & 708 & 604 & 474 & 1786 \\
$\%$ & $39.6 \%$ & $33.8 \%$ & $26.5 \%$ & $100.0 \%$ \\
\hline
\end{tabular}

\section{METODOLOGÍA Y/O INSTRUMENTOS UTILIZADOS}

\section{Instrumentos}

School Refusal Assessment Scale-Revised for Children (SRAS-R-C; Kearney, 2006): La SRASR-C es un instrumento tipo Likert encargada de evaluar el rechazo escolar en población infantil y adolescente. La escala se compone de 24 ítems con 7 tipos de respuesta $(0=$ Nunca; $6=$ Siempre $)$, y cuatro dimensiones subyacentes al rechazo a la escuela. Fl. Evitar la afectividad negativa que provocan los estímulos o situaciones relacionadas con el ámbito escolar (e.g.: ¿Cuántas veces sientes sentimientos negativos hacia la escuela porque tienes miedo a algo relacionado con la escuela (por ejemplo: exámenes, transporte escolar, maestro, alarma de incendio)?); FII. Escapar de la aversión social o situaciones de evaluación (e.g.: ¿Cuántas veces tratas de no ir a la escuela porque te da vergüenza estar delante de otras personas en la escuela?); FIII. Búsqueda de la atención de otras personas significativas (e.g.: ¿Cuántas veces piensas en tus padres o en tu familia cuando estás en la escuela?), y FIV. Búsqueda de refuerzos tangibles fuera del ámbito escolar (e.g.: Cuando no estás en la escuela durante la semana, ¿cuántas veces sales de casa y haces algo divertido?). Gonzálvez et al. (2016), con población española, probaron las propiedades psicométricas del SRAS-R-C, con niveles de fiabilidad adecuados ( $\alpha=$ entre .70 y .87 ).

Child and Adolescent Perfectionism Scale (CAPS; Flett, Hewitt, Boucher, Davidson y Munro, 2000; Flett et al., 2016). La CAPS utilizada en el actual trabajo fue la versión española (Vicent, 2017), la cual se trata de un test tipo Likert de 5 puntos ( 1 = Nada cierto en mí, $5=$ Muy cierto en $\mathrm{mi}$ ) compuesto por 13 ítems, que a su vez lo estructuran 3 dimensiones: Fl. Perfeccionismo Socialmente Prescrito (e.g.: Mi familia espera que yo sea perfecto); Fll. Perfeccionismo Auto Orientado-Esfuerzos (e.g.: Siento que siempre tengo que hacerlo lo mejor posible) y FIII. Perfeccionismo Auto OrientadoCríticas (e.g.: Me enfado incluso si solo hay un error en mi trabajo). Los niveles de fiabilidad fueron aceptables tanto para el total de la escala $(\alpha=.70)$ como para sus tres dimensiones de PSP ( $\alpha=$ .80), PAO-E $(\alpha=.75)$ y PAO-C $(\alpha=.72)$ (Vicent, 2017).

\section{Procedimiento}

En primer lugar, con objeto de solicitar colaboración en el estudio y explicar el objetivo de la investigación, el equipo investigador se entrevistó con los tres equipos directivos de los centros donde se llevó a cabo la administración de los cuestionarios.

En esta entrevista, se destacó la temática, el objetivo y la relevancia del consentimiento informado de los tutores legales de los participantes para poder participar. 
Una vez realizada la entrevista, se llevó a cabo la administración de los cuestionarios en una sesión de aproximadamente 20 minutos. En el inicio de la sesión se explicó la finalidad de la investigación y se destacó que el estudio era voluntario y cada uno de los cuestionarios cumplimentados era anónimo.

\section{Análisis de datos}

Con el objetivo de analizar si existían diferencias estadísticamente significativas en las tres dimensiones de la CAPS (Vicent, 2017; Ortega-Sandoval, 2017) en función del segundo factor del SRAS-R-C (Gonzálvez et al., 2016), se realizaron pruebas $t$ de Student.

Para la interpretación de los resultados se siguieron las indicaciones de Cohen (1988), el cual considera que entre .20 y .49 las puntuaciones son de pequeña magnitud, entre .50 y .79 las puntuaciones son de moderada magnitud, y a partir de .80 las puntuaciones son de elevada magnitud.

Del mismo modo, para determinar si un nivel es alto 0 bajo en escapar de la aversión social 0 situaciones de evaluación se tuvo en cuenta percentiles entre $\geq 75=$ nivel alto, $y \leq 25=$ nivel bajo.

Se utilizó el programa estadístico SPSS 22.0 para realizar los análisis del estudio.

\section{RESULTADOS ALCANZADOS}

Como se puede observar en la Tabla 2, Ios resultados arrojan diferencias estadísticamente significativas de dos de las dimensiones de Perfeccionismo, para adolescentes con altas y bajas puntuaciones en el Factor II del SRAS-R-C, "Escapar de la aversión social o situaciones de evaluación". Estas dos dimensiones son el PAO-E y el PAO-C. No se observan diferencias estadísticamente significativas para el PSP.

Tabla 2.

Diferencias en perfeccionismo en adolescentes con altas y bajas puntuaciones en el FII del SRAS-R-C

\begin{tabular}{cccccccccccc}
\hline Variable & \multicolumn{2}{c}{$\begin{array}{c}\text { Prueba } \\
\text { Levene }\end{array}$} & \multicolumn{3}{c}{$\begin{array}{c}\text { Altas } \\
\text { puntuaciones }\end{array}$} & \multicolumn{2}{c}{$\begin{array}{c}\text { Bajas } \\
\text { puntuaciones }\end{array}$} & \multicolumn{4}{c}{$\begin{array}{c}\text { Significación estadística y } \\
\text { magnitud diferencias }\end{array}$} \\
\hline & $F$ & $p$ & $M$ & $D E$ & $M$ & $D E$ & $t$ & $g . l$. & $p$ & $d$ \\
\hline PSP & 12.90 & $<.001$ & 16.85 & 4.21 & 16.88 & 4.83 & .08 & 971.7 & .930 & - \\
PAO-E & 3.99 & .046 & 13.17 & 3.50 & 13.85 & 3.87 & 2.96 & 987.7 & .003 & .18 \\
PAO-C & 11.88 & .001 & 12.72 & 3.34 & 11.74 & 3.88 & 4.36 & 965.4 & $<.001$ & .27 \\
\hline
\end{tabular}

Nota: Fll SRAS = Escapar de la aversión social 0 situaciones de evaluación: PSP = Perfeccionismo Socialmente Prescrito; PAO-E = Perfeccionismo Auto Orientado Esfuerzos; $\mathrm{PAO}-\mathrm{C}=$ Perfeccionismo Auto Orientado Crítica

La Figura 1 muestra cómo aquellos estudiantes con altos niveles en el escape de la aversión social o situaciones de evaluación obtuvieron puntuaciones más elevadas que sus iguales con bajos niveles del mismo factor del SRAS-R-C, para el PAO-E $(d=.18)$ y el PAO-C $(d=.27)$, siendo estas diferencias de pequeña magnitud. 
Figura 1. Diagrama de barras para las diferencias en puntuaciones de perfeccionismo en estudiantes con altas y bajas puntuaciones en el Factor II del SRAS.

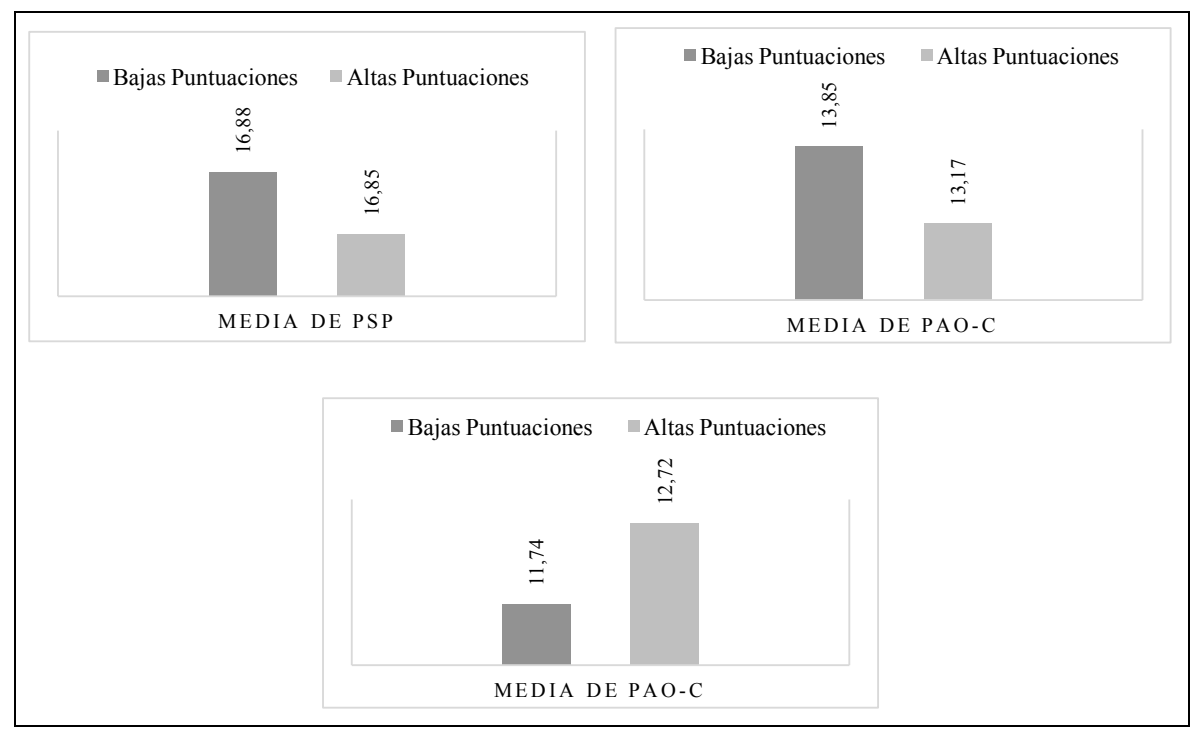

Nota: $^{*}=p<.05 ;{ }^{* *}=p<.01 ;{ }^{* * *}=p<.001$

\section{DISCUSIÓN}

El estudio actual tenía como objetivo observar si existen diferencias significativas en PSP, PAOC y PAO-E en función del alto o bajo rechazo escolar debido a querer escapar de la aversión social o situaciones de evaluación.

Los resultados del trabajo arrojan diferencias estadísticamente significativas, de pequeña magnitud, en PAO-E y PAO-C en el segundo factor del SRAS-R-C. De este modo, los estudiantes con más elevados niveles de rechazo escolar debido a desear evitar la aversión social o diversas circunstancias evaluativas, fueron los que puntuaron significativamente más elevado tanto en PAO-E como en PAO-C.

Cabe destacar que el segundo factor del SRAS-R-C se relaciona con un grupo de niños o adolescentes que rechazan la institución educativa por cuestiones sociales. Es decir, porque no quieren participar en actividades colectivas y sociales tales como deporte, conversaciones entre amigos, ejercicios académicos en grupo, entre otras, así como momentos de evaluación tales como pruebas evaluativas (Kearney, Lemos y Silverman, 2005). Es decir, según Kearney et al. (2006), este grupo de población juvenil se niega a ir al colegio porque este le conlleva a obtener un refuerzo negativo, lo que conforma un descenso de excitación física o un aumento de emociones negativas.

Asimismo, cabe destacar que la dimensión general de PAO compuesta por Hewitt, Flett, Besser, Sherry y McGee (2003) sugiere que se trata de una autoimposición de metas tan elevadas e imposibles de lograr que no alcanzan el realismo, por lo que los sujetos que lo padecen conviven con la tendencia de criticarse severamente si cometen algún error (Vicent, Inglés, Sanmartín, Gonzálvez y García-Fernández, 2017).

En este sentido, estudios que miden las diferencias de PAO en población infantil española demuestran que el alto PAO-C puntúa significativamente más elevado que sus compañeros con bajo 
tanto en afecto negativo como en neuroticismo (Vicent et al., 2019). Es decir, lleva consigo un comportamiento desadaptativo que se caracteriza por sentimientos elevador de inferioridad y culpa si algunas de las expectativas no son cumplidas, lo que desencadena en un malestar subjetivo para los sujetos que lo padecen (Blatt, D’Afflitti y Quinlan, 1976). De ahí que, si un niño con PAO-C siente que en algún momento de evaluación social o académica está fallando podría desencadenar una crisis que origine ese rechazo escolar.

No obstante, Vicent, Inglés, Sanmartín et al. (2019) en su estudio, con población infantil española, afirmaron que el PAO-E obtuvo puntuaciones significativamente más altas en las dimensiones más adaptativas tales como el afecto positivo, amabilidad, conciencia, extraversión y apertura a la experiencia, así como menos elevadas en afecto negativo y neuroticismo. De hecho, otro estudio del mismo equipo, con una muestra etaria similar, corrobora que este tipo de perfeccionismo marca la ausencia de diferencias estadísticamente significativas en cualquiera de las dimensiones de la agresión (hostilidad, ira, agresión física y agresión verbal) (Vicent, Inglés, Gonzálvez et al., 2019). Es, en este sentido importante tener en cuenta la edad y la cultura de los participantes del estudio, así como medir exactamente las mismas variables para poder corroborar los hallazgos.

Gonzálvez et al. (2015) afirmaron que grupos con alto y bajo rechazo escolar debido al segundo factor del SRAS-R-C, mostraron diferencias significativas en PSP, teniendo en cuenta la ansiedad generalizada y social con la que se asocia el factor, según los autores. Por lo que, nuevamente cabe destacar las diferencias culturales y etarias.

Es por ello, que el presente estudio tiene algunas limitaciones. Por un lado, cabe destacar que se trata de un estudio pionero en el ámbito educativo por lo que respecta a la muestra participante en el país de Ecuador, teniendo en cuenta que, desde nuestro conocimiento, no se ha obtenido información previa que examine con exactitud las variables presentes en población ecuatoriana. Sin embargo, sí se han observado estudios como los de Gonzálvez et al. 2015, en población infantil española. Teniendo en cuenta esta tesitura, sería importante replicar el estudio en población infantil ecuatoriana, así como en población adolescente española, con objeto de observar si los hallazgos obtenidos son similares a los del presente estudio.

Del mismo modo, cabría destacar que el trabajo ha sido basado en función de las altas o bajas puntuaciones del segundo factor del SRAS-R-C. Sin embargo, podría ser interesante aumentar el conocimiento en base a las diferencias que pueden presentarse dependiendo del sexo y del curso.

\section{CONCLUSIONES}

Es importante destacar estudios como los de Ingul y Nordahl (2013) cuyos autores examinaron las diferencias de ansiedad social entre grupos de estudiantes de secundaria que asistían diariamente al aula y estudiantes que se ausentaban continuadamente. El segundo de los grupos presentó más niveles de ansiedad social y pánico que el colectivo que solía asistir a la escuela. Por lo que, estudios como el presente son necesarios para determinar que existe una problemática en la que se debe trabajar. Para ello, futuros estudios deberían examinar el diseño y planteamiento de programas de prevención y/o reducción tanto de rechazo y ansiedad escolar, como de perfeccionismo en todas sus facetas, teniendo en cuenta además que los niños con problemas de integración tienden además a presentar una baja autoestima social (Cava y Musitu, 2001), lo que complicaría cada vez más el problema. De ahí la necesidad de ampliar el campo de estudio en diversas culturas, así como amparar su detección y prevención.

\section{REFERENCIAS BIBLIOGRÁFICAS}

Blatt, S. J., D' Afflitti, J. P. y Quinlan, D. M. (1976). Experiences of depres-sion in normal young adults. Journal of Abnormal Psychology, 85, 383-389. doi: 10.1037/0021-843X.85.4.383 
Broadwin, I. T. (1932). A contribution to the study of truancy. American Journal of Orthopsychiatry, 2 (3), 253-259. doi: 10.1111/j.1939-0025.1932.tb05183.x

Burns, D. D. (1980). The Perfectionist's script for self-defeat. Psychology today. 14 (6), 34-52.

Cava, M. J., y Musitu, G. (2001). Autoestima y percepción del clima escolar en niños con problemas de integración social en el aula. Revista de Psicología General y Aplicada, 54(2), 297-311.

Cohen, J. (1988). Statistical power analysis for the behavioral sciences. Hillsdale, NJ: Erlbaum.

Flett, G. L. y Hewitt, P. L. (2005). The perils of perfectionism in sports and exercise. Current Directions in Psychological Science, 14, 14- 18

Flett, G. L., Hewitt, P. L., Besser, A., Su. C., Vaillancourt, T., Boucher, D., ... Gale, O. (2016). The child-adolescent perfectionism scale: development, psychometric properties, and associations with stress, distress, and psychiatric symptoms. Journal of Psychoeducational Assessment, 34 (7), 634-652. doi: 10.1177/07342829166651381

Flett, G. L., Hewitt, P. L., Boucher, D. J., Davidson, L. A. y Munro, Y. (2000). The Child-Adolescent Perfectionism Scale: development, validation, and association with adjustment. Manuscrito inédito, York University, Toronto, Ontario, Canadá.

García-Fernández, J. M., Gonzálvez, C., Vicent, M., Delgado, B. y Gómez-Núñez, M. I. (2016). Revisión bibliométrica del rechazo escolar: perspectivas de investigación y su análisis [Bibliometric review of school refusal: research perspectives and their analysis]. Educatio Siglo XXI, 34 (1), 71-92. doi: 10.6018/j/252541

Gonzálvez, C., Inglés, C. J., Kearney, C.A., Vicent, M., Sanmartín, R. y García-Fernández, J. M. (2016). School Refusal Assessment Scale-Revised: Factorial invariance and latent means differences across gender and age in Spanish children. Frontiers in Psychology, 7, 1-10. doi: 10.3389/fpsyg.2016.02011

Gonzálvez, C., Sanmartín, R., García-Fernández, J. M., Inglés, C. J. y Vicent, M. (2016). Perfeccionismo socialmente prescrito como predictor del alto rechazo a la escuela. International Journal of Developmental and Educational Psychology, 1(1), 25-31. doi: 10.1760/ijodaep.2016.n.1.v1.185

Gonzálvez, C., Vicent, M., Inglés, C. J., García-Fernández, J. M. y Martínez-Monteagudo, M. C. (2015). Diferencias en perfeccionismo socialmente prescrito en función del rechazo escolar. International Journal of Developmental and Educational Psychology, 1 (1), 455- 462. doi: 10.17060/ijodaep.2015.n1.v1.47

Estes, H. R., Haylett, C. H. y Johnson, A. M. (1956), Separation anxiety. American Journal of Psychotherapy, 10, 682-695.

Hamachek, D. (1978). Psychodynamics of normal and neurotic perfectionism. Psychology: $A$ Journal of Human Behavior, 15 (1), 27-33.

Hewitt, P. L., Flett, G. L., Besser, A., Sherry, S. B. y McGee, B. (2003). Perfectionism is multidimensional: a reply to Shafran, Cooper \& Fairburn (2002). Behaviour Research and Therapy, 41, 1221-1236. doi: 10.1016/S0005-9767(03)00021-4

Inglés, C. J., García-Fernández, J. M., Vicent, M., Gonzálvez, C. y Sanmartín, R. (2016). Profiles of perfectionism and school anxiety: a review of the $2 \times 2$ model of dispositional perfectionism in child population. Frontiers in Psychology, 7, 1403. doi: 10.3389/fpsyg.2016.01403

Ingul, J. M. y Nordahl, H. M. (2013). Anxiety as a risk factor for school absenteeism: What differentiates anxious school-attenders from non-attenders?. Annals of General Psychiatry, 12 (25), 1-9. doi: 10.1186/1744-859X-12-25

Johnson, A. M., Falstein E. I., Szurek S. A. y Svendsen, M. (1941). School phobia. American Journal of Orthopsychiatry,11(4), 702-711. doi: 10.1111/j.1939-0025.1941.tb05860.x 
Kearney, C. A. (2002). Identifying the function of school refusal behavior: A revision of the School Refusal Assessment Scale. Journal of Psychopathology and Behavioral Assessment, 24 (4), 235- 245. doi: 10.1023/A:1020774932043

Kearney, C. A. (2006). Confirmatory factor analysis of the School Refusal Assessment Scale-Revised: Child and parent versions. Journal of Psychopathology and Behavioral Assessment, 28(3), 139-144. doi: 10.1007/s10862-005-9005-6

Kearney, C. A. y Bates, M. (2005). Addressing School Refusal Behavior: Suggestions for Frontline Professionals. Children \& Schools, 27 (4), 207-216. doi: 10.1093/cs/27.4.207

Kearney, C. A., Lemos, A. y Silverman, J. (2006). School refusal behavior. En R. B. Mennuti, A. Freeman, y R.W. Christner (Eds.), Cognitive-behavioral interventions in educational settings: $A$ handbook for practice (pp. 89-105). New york: Brunner-Routledge.

Ortega- Sandoval, V. N. (2019). Rechazo escolar del alumnado de Bachillerato en la ciudad de Quito (Tesis doctoral). Universidad de Alicante, Alicante, España.

Rice, K. G., Bair, C. J., Castro, J. R., Cohen, B. N. y Hood, C. A. (2003). Meanings of perfectionism: a quantitative and qualitative analysis. Journal of Cognitive Psychotherapy, 17, 39-58. doi: 10.1891/jcop.17.1.39.58266

Slade, P. D. y Owens, R. G. (1998). A dual process model of perfectionism based on reinforcement theory. Behavior Modification, 22, 372-390. doi: 10.1177/01454455980223010

Stoeber, J. y Otto, K. (2006). Positive conceptions of perfectionism: approaches, evidence, challenges. Personality and Social Psychology Review, 10, 295-319. doi: 10.1207/s15327957pspr1004_2

Vicent, M. (2017). Estudio del perfeccionismo y su relación con variables psicoeducativas en la infancia tardía [Study of perfectionism and its relationship with psycho-educational variables during late childhood] (Tesis doctoral). Universidad de Alicante, Alicante, España.

Vicent, M., Inglés, C. J., Gonzálvez, C., Sanmartín, R., Aparicio-Flores, M. P., y García-Fernández, J. M. (2019). Self-critiocism, strivings and aggressive behavior in Spanish children: the two sides of self-oriented perfectionism. The Spanish Journal of Psychology, 22 (29), 1-8. doi: $10.1017 /$ sjp.2019.29

Vicent, M., Inglés, C. J., Sanmartín, R., Gonzálvez, C., Aparicio-Flores, M. P. y García-Fernández, J. M. (2019). Clarifying the two facets of Self-Oriented Perfectionism: influences on affect and the Big Five traits of personality in children. Anales de psicología, 35(2), 280-289. doi: 10.6018/analesps.35.2.331441

Vicent, M., Inglés, C. J, Sanmartín, R., Gonzálvez, C. y García-Fernández, J. M. (2017). Perfectionism and aggression: identifying risk profiles in children. Personality and Individual Differences, 112, 106-112. https://doi.org/10.1016/j.paid.2017.02.061 
